\title{
Robotic Transcranial Doppler in Non-Traumatic Subarachnoid Hemorrhage: A Safety and Efficacy Study
}

\section{Kevin Clare}

New York Medical College School of Medicine

\section{Alan Stein}

Westchester Medical Center at New York Medical College

\section{Nitesh Damodara}

Westchester Medical Center at New York Medical College

\section{Eric Feldstein}

Westchester Medical Center at New York Medical College

\section{Hussein Alshammari}

Westchester Medical Center at New York Medical College

\section{Syed Ali}

Westchester Medical Center at New York Medical College

Christeena Kurian

Westchester Medical Center at New York Medical College

Jon Rosenberg

Westchester Medical Center at New York Medical College

Andrew Bauerschmidt

Westchester Medical Center at New York Medical College

\section{Gurmeen Kaur}

Westchester Medical Center at New York Medical College Justin Santarelli

Westchester Medical Center at New York Medical College

Robert Hamilton

NovaSignal Corp

\section{Stephan Mayer}

Westchester Medical Center at New York Medical College

Chirag D. Gandhi

Westchester Medical Center at New York Medical College

Fawaz Al-Mufti ( $\square$ fawaz.al-mufti@wmchealth.org )

Westchester Medical Center at New York Medical College 


\section{Research Article}

Keywords: Subarachnoid hemorrhage, Transcranial doppler, Vasospasm

Posted Date: September 28th, 2021

DOl: https://doi.org/10.21203/rs.3.rs-923453/v1

License: (c) (i) This work is licensed under a Creative Commons Attribution 4.0 International License. Read Full License 
Robotic Transcranial Doppler in Non-Traumatic Subarachnoid Hemorrhage: A

Safety and Efficacy Study

Kevin Clare, BE ${ }^{\mathrm{a}}$; Alan Stein, $\mathrm{MD}^{\mathrm{b}}$; Nitesh Damodara, $\mathrm{MD}^{\mathrm{b}}$; Eric Feldstein, $\mathrm{MD}^{\mathrm{b}}$, Hussein Alshammari, $\mathrm{MD}^{\mathrm{c}}$; Syed Ali, $\mathrm{MD}^{\mathrm{c}}$; Christeena Kurian, $\mathrm{MD}^{\mathrm{c}}$; Jon Rosenberg, $M D^{b, c} ;$ Andrew Bauerschmidt, MD ${ }^{b, c}$; Gurmeen Kaur, MD ${ }^{b, c}$; Justin Santarelli MD Robert Hamilton, PhD ${ }^{\mathrm{d}}$; Stephan Mayer, MD; Chirag D. Gandhi, MD ${ }^{\mathrm{b}}$; Fawaz Al-Mufti, $M D^{b, c}$

a. New York Medical College School of Medicine, Valhalla, New York, USA.

b. Department of Neurosurgery, Westchester Medical Center at New York Medical College, Valhalla, New York, USA.

c.Department of Neurology, Westchester Medical Center at New York Medical College, Valhalla, New York, USA.

d.NovaSignal Corp., Los Angeles, CA, USA

Word Count: 1970

Number of Figures and Tables: 1 Table; 2 Figures

Corresponding author:

Fawaz Al-Mufti, MD

Westchester Medical Center at New York Medical College 100 Woods Road, Macy Pavilion 1331,

Valhalla, NY 10595

Phone: 914-493-5216

Email: fawaz.al-mufti@wmchealth.org

Running Title: Safety and Efficacy NovaGuide TCD 
Key Words: Subarachnoid hemorrhage, Transcranial doppler, Vasospasm

\begin{abstract}
Introduction: Delayed cerebral ischemia (DCl) secondary to vasospasm is a determinate of outcomes following non-traumatic subarachnoid hemorrhage (SAH).

SAH patients are monitored using transcranial doppler (TCD) to measure cerebral blood flow velocities (CBFv). However, the accuracy and precision of manually acquired TCD can be operator dependent. The NovaGuide robotic TCD system attempts to standardize acquisition. This investigation evaluated the safety and efficacy of the NovaGuide system in SAH patients in a Neuro ICU.
\end{abstract}

Methods: We retrospectively identified 48 NovaGuide scans conducted on SAH patients. Mean and maximum middle cerebral artery (MCA) CBFv were obtained from the NovaGuide and the level of agreement between CBFv and computed tomography angiography (CTA) for vasospasm was determined. Safety of NovaGuide acquisition of CBFv was evaluated based on number of complications with central venous lines (CVL) and external ventricular drains (EVD)

Results: There was significant agreement between the NovaGuide and CTA (Cohen's Kappa $=0.74)$ when maximum MCA CBFv $\geq 120 \mathrm{~cm} / \mathrm{s}$ was the threshold for vasospasm. 27/48 scans were carried out with CVLs and EVDs present without negative outcomes.

Conclusions: The lack of adverse events associated with EVDs/CVLs and the strong congruence between maximal MCA CBFv and CTA illustrates the diagnostic utility of the NovaGuide. 


\section{INTRODUCTION}

Each year the United States has 30,000 cases of subarachnoid hemorrhage $(\mathrm{SAH})$, which is consistent with the global incidence rate and represents a significant cause of stroke-related death and disability. ${ }^{1}$ Vasospasm is the narrowing of the cerebral arteries and is a common complication of SAH that can lead to delayed cerebral ischemia $(\mathrm{DCl})$, with $\mathrm{DCl}$ leading to poor patient outcomes and is associated with morbidity and mortality following SAH..$^{2,3}$ Digital subtraction angiography (DSA), and increasingly computer tomography angiography (CTA) are considered the gold standard for determination of cerebral vasospasm as it allows for clear visualization of vasculature. ${ }^{4}$ However, repeated imaging from DSA and CTA does not come without risks especially in patients with renal comorbidities. Transcranial doppler (TCD) is a non-invasive bedside modality to rapidly assess vasospasm or stenosis of cerebral arteries following SAH.

TCD offers a safe, relatively inexpensive, and repeatable alternative to CTA to measure cerebral blood flow velocity (CBFv) by insonating proximal cerebral arteries, thus enabling the identification of vasospasm and ischemia. ${ }^{5}$ In addition, there are a plethora of potential utilizations of TCD in the neurocritical care setting, such as traumatic brain injury, non-invasive intracranial pressure (ICP) monitoring, large vessel occlusions, sickle cell disease, cerebral malaria, periprocedural monitoring, and even brain stem death. ${ }^{6}$ Furthermore, TCD monitoring is also able to predict microemboli burden and as such has shown efficacy for stroke risk stratification after blunt cerebrovascular injury to internal cerebral arteries. ${ }^{7}$ Specific thresholds of mean 
cerebral blood flow have been defined to create criteria for the diagnosis of vasospasm based on TCD readings. ${ }^{8}$ However, manual capture of TCD requires a trained sonographer which limits its availability to specific institutions and results in TCD being highly operator dependent. Technicians often have difficulty in finding adequate acoustic windows for ultrasound, leading to vessels not being properly insonated or unsuccessful monitoring attributable to movement of the probe during the procedure..$^{9,10}$ Moreover, studies have demonstrated as much as $22.1 \mathrm{~cm} / \mathrm{s}$ variability between experienced operators. ${ }^{11}$

The NovaGuide (NovaGuide Intelligent Ultrasound, NovaSignal Corp., Los Angeles CA USA) aims to expedite, standardize, and enhance TCD blood flow measurements through combining artificial intelligence and autonomous acquisition that minimizes the need for a trained operator. The NovaGuide system uses a headset that automatically moves the transducer, eliminating variability between users. This allows the system to be used without a trained sonographer while also providing consistent results. We sought to evaluate the safety and validity of the NovaGuide in patients with SAH.

\section{METHODS}

This research was conducted at Westchester Medical Center and was approved by New York Medical College Institutional Review Board and the Clinical Research Institute at Westchester Medical Center. All methods were performed in accordance with the relevant guidelines. The data will be shared by the principal investigator (F.A.M.) upon reasonable request. We retrospectively evaluated our SAH database to 
identify patients who underwent NovaGuide imaging between March $1^{\text {st }}, 2020$ to April $30^{\text {th }}, 2020$. We collected demographic data, admission clinical and radiologic features, and whether the patient had an external ventricular drain (EVD) or central venous line $(C V L)$ at the time of their TCD examinations. Informed consent was obtained from all subjects and/or their legal guardians. Our management protocol calls for CTA on SAH days 4, 7 and 10-12 (with day 0 being the calendar day of SAH onset). NovaGuide TCD scans were acquired on the same day as the CTAs. The degree of vasospasm in the proximal M1 segment of the middle cerebral arteries was graded as absent, mild, moderate, or severe by a neuroradiologist in a standard clinical radiology report, who was blinded to the TCD results.

To assess for complications associated with the use of the NovaGuide device, we analyzed electronic medical records for nursing documentation of any EVD or CVL disruptions or removals. From the TCD readouts, we collected the maximal and average mean blood flow velocities and pulsatility indices $(\mathrm{PI})$ from the left and right middle cerebral arteries. Average mean velocity $\geq 86 \mathrm{~cm} / \mathrm{s}$ or a maximum mean flow velocity $\geq 120 \mathrm{~cm} / \mathrm{second}$ were used as thresholds for defining vasospasm of the middle cerebral artery. After categorizing the velocities, we compared the level of agreement between the NovaGuide TCD measured velocities and CTA reports using a Cohen's Kappa test. All statistics were run using R-studio and were considered significant when $\mathrm{P}<0.05$.

TCD data was collected using the NovaGuide System (NovaGuide Intelligent Ultrasound, NovaSignal Corp., Los Angeles CA USA) which uses $2 \mathrm{MHz}$ bilateral probes contained within a five degree of freedom robotic mechanism (Figure 1). The 
system is setup on the patient in the supine position $\left(0^{\circ}\right.$ to $45^{\circ}$ head of bed angle) with the patient's head resting in the system's head cradle. Once the patient is positioned in the NovaGuide head cradle, the healthcare provider gently engages the robotic probes onto the patient's temporal region. The system uses machine vision to co-register the patient with the robot using specially designed fiducials. After the registration, signal acquisition includes two steps, transtemporal window identification, and MCA signal optimization with no operator involvement after setup. NovaGuide uses signal quality assessment (SQA) as a rapid means of evaluating signals in real time with segments of data between $350 \mathrm{~ms}-500 \mathrm{~ms} .{ }^{12,13}$

\section{RESULTS}

Our study population consisted of twelve patients with two males and ten females with an average age of 63.5 years. From the identified cohort, $50 \%$ had radiologically confirmed vasospasm in the cerebral vasculature including the branches of the middle cerebral arteries $(66 \%)$, the posterior cerebral arteries $(17 \%)$, and the vertebral arteries (17\%) with a mean Hunt Hess score of 2.7. At time of discharge, the average mRS was 3.0 with four patients discharge home, three to in-patient rehabilitation, three to skilled nursing facilities, one to long term acute care facility, and one deceased. Seven of the twelve subjects had an external ventricular drain and central venous line at the time of NovaGuide acquisition. Those seven patients underwent a total of 27 acquisitions using the autonomous robotic device to acquire mean and maximum cerebral blood flow velocities of the middle cerebral arteries. There were no complication associated with 
the patient's EVD or CVL caused by the motion of the NovaGuide insonators during velocity acquisition (Table 1). 


\section{Table 1: Patient characteristics and outcomes}

\begin{tabular}{|c|c|}
\hline Characteristic: & Values: \\
\hline Median age - yr (IQR) & $63.5(55-70.25)$ \\
\hline Female - no. (\%) & $10(84)$ \\
\hline Radiologically confirmed vasospasm - no. (\%) & $6(50)$ \\
\hline \multicolumn{2}{|l|}{ Locations } \\
\hline MCA - no. (\%) & $4(66)$ \\
\hline PCA - no. (\%) & $1(17)$ \\
\hline$A C A-$ no. (\%) & $1(17)$ \\
\hline \multicolumn{2}{|l|}{ Outcomes: } \\
\hline Hunt Hess grade & $2.7(1-4)$ \\
\hline $\mathrm{mRS}$ at discharge & $3.0(1-6)$ \\
\hline \multicolumn{2}{|l|}{ Discharged to: } \\
\hline Home & 4 \\
\hline IP Rehab & 3 \\
\hline SNF & 3 \\
\hline LTAC & 1 \\
\hline Deceased & 1 \\
\hline \multicolumn{2}{|l|}{ NovaGuide scans and EVD/CVL complications: } \\
\hline NovaGuide scans for CTA confirmed MCA vasospasm - no. (\%) & $17(35.4)$ \\
\hline Patients with external ventricular drain \& central venous line - no. (\%) & $7(58.4)$ \\
\hline Total number of NovaGuide scans in EVD \& CVL patients - no. (\%) & $27(56.2)$ \\
\hline EVD/CVL complications - no. (\%) & $0(0)$ \\
\hline
\end{tabular}

Abbreviations: In-patient rehabilitation (IP Rehab); Skilled nursing facility (SNF); Long term acute care (LTAC). External ventricular drain (EVD); Central venous line (CVL). 



Figure 1: NovaGuide System - five degree of freedom robotic TCD system. [Original image created by manuscript author Dr. Robert Hamilton of NovaSignal]. 




Figure 2: a) Confusion matrices for the outcome of using mean CBFv $\geq 86 \mathrm{~cm} / \mathrm{s}$. b) Maximum CBFv $\geq 120 \mathrm{~cm} / \mathrm{s}$ as a diagnostic metric for determination of vasospasm. c) Parameters quantifying the performance of these metrics. 
Using seventeen $(m=17)$ NovaGuide readings in patients $(n=4)$ with confirmed vasospasm in the MCAs and sixteen $(m=16)$ TCD results from patients with no vasospasm $(n=4)$ the thresholds of the NovaGuide acquired mean CBFv $\geq 86 \mathrm{~cm} / \mathrm{s}$ or maximum $C B F v \geq 120 \mathrm{~cm} / \mathrm{s}$ were evaluated for their value as diagnostic criteria. When the mean cerebral blood flow velocity was used as the parameter to predict the presence of MCA vasospasm, no true positive or false positive events occurred (Figure 2a). Therefore, the Cohen's Kappa value, sensitivity, positive predictive value (PPV), positive and negative likelihood ratios could not be determined (Figure 2c). However, when the max mean cerebral blood flow velocity $\geq 120 \mathrm{~cm} / \mathrm{s}$ was used as the threshold for vasospasm all aspects of the confusion matrix were accounted for (Figure 2b). Calculation of the Cohen's Kappa value for the level of agreement between the NovaGuide and the CTA demonstrated substantial inter-observer agreement between the two modalities with a Cohen's kappa value of $0.74(P<0.001)$. This parameter also had a strong positive and negative predictive value able to indicate the presence of vasospasm in $84 \%$ of positive cases and the absence of vasospasm in $90 \%$ of negative cases (Figure 2c). Moreover, using maximum CBFv $\geq 120 \mathrm{~cm} / \mathrm{s}$ for diagnostic criteria of vasospasm it yielded a positive likelihood ratio $(\mathrm{LR}+)$ of 8.75 suggesting that a positive result using this metric means that the patient has $\sim 40 \%$ increased probability of having vasospasm post positive NovaGuide scan (Figure 2c).

\section{Discussion}

The present investigation aimed to evaluate the safety and efficacy of the utilization of the NovaGuide system in the setting of a neurocritical care unit to assess 
for the presents of vasospasm in patients with acute subarachnoid hemorrhage. One of the prominent safety concerns regarding the NovaGuide device is the autonomous guidance of the insonators on the scalp and the risk this motion may pose to external CVL or EVD. We experience no complications associated with the autonomous motion on the EVDs or CVLs in the 27 scans performed. Additionally, we compared the mean and maximum CBFv for the MCA obtained by NovaGuide against CTA confirmed vasospasm. The diagnostic threshold of a maximum CBFv $\geq 120 \mathrm{~cm} / \mathrm{s}$ produced substantial inter-observer agreement between the NovaGuide and CTA outcomes as well as displayed high sensitivity, specificity, and positive likelihood ratio.

Based on current literature, the consensus among publications is that a normal mean CBFv is below $85 \mathrm{~cm} / \mathrm{s}$ with any value above this cut-off indicating abnormal flow and vessel stenosis. Specifically for vasospasms, a velocity between $86 \mathrm{~cm} / \mathrm{s}$ and 120 $\mathrm{cm} / \mathrm{s}$ is thought to indicate mild vasospasm, velocities from $120-200 \mathrm{~cm} / \mathrm{s}$ as moderate to severe spasm, and greater than $200 \mathrm{~cm} / \mathrm{s}$ to be critical. ${ }^{14,15}$ Thus, we first investigated the mean CBFv as a diagnostic criterion for detecting vasospasm using the NovaGuide acquired flow velocities. Surprisingly, there were no true positives detected when using this criterion. It is highly unlikely that this outcome is due to the NovaGuide system as it is an effective tool for acquiring MCA CBFv as documented in the study by O'Brien et al. where the NovaGuide matched the efficacy in MCA signal acquisition of a registered vascular technician (RVT) on 86 healthy subjects with a mean velocity accuracy of 99.7\% (95\% Cl: $97.7 \%$ - 101.7\%) with respect to the RVT. This investigation also showed a mean time to signal acquisition of $74.4 \mathrm{~s}(95 \% \mathrm{Cl}: 60 \mathrm{~s}-90 \mathrm{~s})$ and a nowindow rate of $3.5 \%$ (RVT no-window rate was $4.1 \%) .{ }^{16}$ However, this outcome might 
be attributed to the age of our patient population as previous studies have documented the decline of mean CBFv with age. ${ }^{17-19}$ Thus, the lack of results using the mean CBFv as diagnostic criteria may reflect the fact that in our patient population the baseline mean CBFv was low. Therefore, when vasospasm did occur as documented by angiography, the rise in CBFv was not great enough to reach or surpass threshold.

Interestingly, when we applied the mean CBFv threshold for mild vasospasm to the maximum CBFv (Max CBFv $\geq 120 \mathrm{~cm} / \mathrm{s}$ ) from the NovaGuide data we obtained a performance comparable to previous publications. A meta-analysis of 26 studies comparing TCD against angiography found that utilizing the criteria of mean CBFv $>120 \mathrm{~cm} / \mathrm{s}$ TCD had a sensitivity of $67 \%$ (48\%-87\%), specificity of $99 \%(99 \%-100 \%)$, positive predictive value of $97 \%$ (95\%-98\%), negative predictive value of $78 \%$ (65\%$91 \%)$, positive likelihood ratio of 17 (5-56) ,and negative likelihood ratio of 0.4 (0.20.7). ${ }^{14}$ Our metrics of sensitivity (83\%), negative predictive value $(90 \%)$, and positive likelihood ratio (8.75) are contained within these reported ranges. Additionally, other groups have reported specificities consistent with our results. ${ }^{6,20}$ Taken together, this suggests that maximum $C B F v \geq 120 \mathrm{~cm} / \mathrm{s}$ may have some diagnostic value in determining the presence of MCA vasospasm.

\section{CONCLUSION}

Although this study yields valuable insight into the usage of the NovaGuide system for determination of cerebral vasospasm in acute $\mathrm{SAH}$, this study is not without limitations. Mainly, the sample size of the current investigation is small with safety being evaluated in seven patients and the detection of MCA vasospasm in four. The low 
number of patients for safety however is partially offset by the total of 27 individual scans performed. Thus, additional work is necessary to substantiate our claim of the safety of the NovaGuide device as well as the efficacy of using maximum CBFv $\geq$ $120 \mathrm{~cm} / \mathrm{s}$ as a diagnostic threshold for MCA vasospasm. Future studies will focus on enrolling additional patients as well as evaluating the relationship between ICP and the Pulsatility Index (PI). 


\section{Disclosure:}

Dr. Robert Hamilton is the Co-Founder \& Chief Scientific Officer of NovaSignal. Other authors have nothing to disclose

\section{Author Contributions:}

F.A.M, C.D.G, and S.M. designed the research; K.C, A.S, and N.D. collected and analyzed the data. K.C, H.A, E.F, S.A, C.K, J.R, A.B, G.K, J.S, R.H, S.M, C.D.G, and F.A.M contributed significantly to data interpretation, discussing the results, and writing the manuscript.

\section{Data Availability Statement:}

The data that support the findings of this study are available from the corresponding author upon reasonable request.

Funding: This research did not receive any specific grant from funding agencies in the public, commercial, or not-for-profit sectors. 


\section{References}

1. Etminan N, Chang H-S, Hackenberg K, et al. Worldwide Incidence of Aneurysmal Subarachnoid Hemorrhage According to Region, Time Period, Blood Pressure, and Smoking Prevalence in the Population: A Systematic Review and Meta-analysis. JAMA Neurol. 2019;76(5):588-597. doi:10.1001/jamaneurol.2019.0006

2. Vergouwen MDI, Vermeulen M, van Gijn J, et al. Definition of delayed cerebral ischemia after aneurysmal subarachnoid hemorrhage as an outcome event in clinical trials and observational studies: proposal of a multidisciplinary research group. Stroke. 2010;41(10):2391-2395. doi:10.1161/STROKEAHA.110.589275

3. Chang JJ, Triano M, Corbin MJ, et al. Transcranial Doppler velocity and associations with delayed cerebral ischemia in aneurysmal subarachnoid Hemorrhage. J Neurol Sci. 2020;415:116934. doi:10.1016/j.jns.2020.116934

4. Staals $\varnothing \mathrm{JM}$, Edsen T, Romner B, Olsen NV. Transcranial Doppler velocimetry in aneurysmal subarachnoid haemorrhage: intra- and interobserver agreement and relation to angiographic vasospasm and mortality. BJA Br J Anaesth. 2013;110(4):577-585. doi:10.1093/bja/aes458

5. Sharma S, Lubrica RJ, Song M, Vandse R, Boling W, Pillai P. The Role of Transcranial Doppler in Cerebral Vasospasm: A Literature Review. Acta Neurochir Suppl. 2020;127:201-205. doi:10.1007/978-3-030-04615-6_32

6. Moppett IK, Mahajan RP. Transcranial Doppler ultrasonography in anaesthesia and intensive care. Br J Anaesth. 2004;93(5):710-724. doi:10.1093/bja/aeh205

7. Bonow RH, Witt CE, Mosher BP, et al. Transcranial Doppler Microemboli Monitoring for Stroke Risk Stratification in Blunt Cerebrovascular Injury. Crit Care Med. 2017;45(10):e1011-e1017. doi:10.1097/CCM.0000000000002549

8. Samagh N, Bhagat $\mathrm{H}$, Jangra K. Monitoring cerebral vasospasm: How much can we rely on transcranial Doppler. J Anaesthesiol Clin Pharmacol. 2019;35(1):12-18. doi:10.4103/joacp.JOACP_192_17

9. Kaczynski J, Home R, Shields K, et al. Reproducibility of Transcranial Doppler ultrasound in the middle cerebral artery. Cardiovasc Ultrasound. 2018;16(1):15. doi:10.1186/s12947-018-0133-z

10. Corriveau MM, Johnston KW. Interobserver variability of carotid Doppler peak velocity measurements among technologists in an ICAVL-accredited vascular laboratory. J Vasc Surg. 2004;39(4):735-741. doi:10.1016/j.jvs.2003.12.017

11. McMahon CJ, Mcdermott P, Horsfall D, Selvarajah JR, King AT, Vail A. The reproducibility of transcranial Doppler middle cerebral artery velocity measurements: 
Implications for clinical practice. Br J Neurosurg. 2007;21(1):21-27.

doi:10.1080/02688690701210539

12. O'Brien M, Ranjbaran M, Thorpe S, et al. Quantitative TCD Signal Quality Assessment. Presented at the: European Society of Neurosonology and Cerebral Hemodynamics; 2018; Prague, CZ.

13. Ranjbaran M, O'Brien M, Nie Z, Radhakrishnan S, Hamilton R. Real-Time, Expert Equivalent, Quantitative TCD Signal Quality Assessment (SQA) Using Short Signal Segments. Presented at the: 24th Meeting of the European Society of Neurosonology and Cerebral Hemodynamics; 2019; Linz, Austria.

14. Lysakowski Christopher, Walder Bernhard, Costanza Michael C., Tramèr Martin R. Transcranial Doppler Versus Angiography in Patients With Vasospasm due to a Ruptured Cerebral Aneurysm. Stroke. 2001;32(10):2292-2298.

doi:10.1161/hs1001.097108

15. Kassab MY, Majid A, Farooq MU, et al. Transcranial Doppler: An Introduction for Primary Care Physicians. J Am Board Fam Med. 2007;20(1):65-71. doi:10.3122/jabfm.2007.01.060128

16. O'Brien M, Ranjbaran M, Nie Z, Scheidt M, Radhakrishnan S, Hamilton R. Fully Automated Transcranial Doppler Ultrasound for Cerebral Blood Flow Measurements in the Middle Cerebral Artery. Presented at the: International Symposium on Intracranial Pressure and Neuromonitoring; 2019; Leuven, Belgium.

17. Droste DW, Harders AG, Rastogi E. A transcranial Doppler study of blood flow velocity in the middle cerebral arteries performed at rest and during mental activities. Stroke. 1989;20(8):1005-1011. doi:10.1161/01.str.20.8.1005

18. Krejza J, Mariak Z, Walecki J, Szydlik P, Lewko J, Ustymowicz A. Transcranial color Doppler sonography of basal cerebral arteries in 182 healthy subjects: age and sex variability and normal reference values for blood flow parameters. AJR Am J Roentgenol. 1999;172(1):213-218. doi:10.2214/ajr.172.1.9888770

19. Demirkaya S, Uluc K, Bek S, Vural O. Normal blood flow velocities of basal cerebral arteries decrease with advancing age: a transcranial Doppler sonography study. Tohoku J Exp Med. 2008;214(2):145-149. doi:10.1620/tjem.214.145

20. Sloan MA, Haley EC, Kassell NF, et al. Sensitivity and specificity of transcranial Doppler ultrasonography in the diagnosis of vasospasm following subarachnoid hemorrhage. Neurology. 1989;39(11):1514-1518. doi:10.1212/wnl.39.11.1514 


\section{Figure Legend}

Figure 1: NovaGuide System - five degree of freedom robotic TCD system. [Original image created by manuscript author Dr. Robert Hamilton of NovaSignal].

Figure 2: a) Confusion matrices for the outcome of using mean $C B F v \geq 86 \mathrm{~cm} / \mathrm{s}$. b) Maximum CBFv $\geq 120 \mathrm{~cm} / \mathrm{s}$ as a diagnostic metric for determination of vasospasm. c) Parameters quantifying the performance of these metrics. 